\title{
CONGENITAL INTESTINAL MALROTATION IN ADULT PATIENTS
}

\author{
Firas Jabir $^{\circledR}$, Issam Merdan*, Wissam Saoud ${ }^{\#} \&$ Hamed Laftah^ $^{\wedge}$ \\ ${ }^{\circledR}$ MBChB, FIBMS General Surgery, FIBMS GIT \& Hepatobiliary Surgery, FACS, Al Sadr Teaching \\ Hospital, Basrah. "MBChB, FIBMS, CABMS, Professor of Surgery, Department of Surgery, College of \\ Medicine, University of Basrah. "MBChB, CABMS, FACS, FIBMS GIT \& Hepatobiliary Surgery, \\ FACS, Al Salam Teaching Hospital, Mosul, Iraq. MBChB, FIBMS, General Medicine, FIBMS GIT \& \\ Hepatobiliary Medicine, Al Fayhaa General Hospital, Basrah, IRAQ.
}

\begin{abstract}
Congenital intestinal malrotation is an impaired embryological development of the gut causing incomplete rotation and attachment of the intestines to the abdominal wall.

Three cases with congenital intestinal malrotation in adult patients were reviewed regarding their presentation, diagnosis and management. Two cases presented as emergency and the other one was incidental. Signs and symptoms of intestinal obstruction were the dominant presentation. A contrast computer tomography and upper gastrointestinal series confirmed the diagnosis of these cases. Ladd's procedure was done in two cases.

In conclusion, Intestinal malrotation should be considered as a cause of intestinal obstruction in all age groups. A contrast computer tomography is the best way to reveal this malformation. Surgical treatment is the greatest choice to relieve symptoms.

Key words: Intestinal malrotation, congenital, surgery, conservative, adults
\end{abstract}

\section{Introduction}

Tmpaired embryological development of 1 the gut due to congenital intestinal malrotation can cause deficient rotation with fixation of the intestines to the abdominal wall ${ }^{1}$. During the sixth week of fetal development, the midgut grows too rapidly to be accommodated in the abdominal cavity and therefore prolepses into the umbilical cord. Between the tenth and twelfth weeks, the midgut returns to the abdominal cavity undergoing a 270degree counterclockwise rotation around the superior mesenteric artery. The fulfillment of the third embryonic rotation includes the traversing of the duodenum to the left side of the abdomen forming the ligaments of Treitz, and also the migration of the ileo-caecal junction to the lower right abdominal quadrant. The fixation of the full-length bowel is complete during the twelfth week ${ }^{2}$.
The inadequate fixation of the bowel alongside remaining embryonic fibrous adhesions, the Ladd's bands ${ }^{3}$, may give rise to a variety of intestinal malfunctions. In the worst case scenario, malrotation may develop into a midgut volvulus with torsion causing high risk of ischemia and necrosis of parts of the intestine supplied by the superior mesenteric artery. This life-threatening condition is well known among pediatric surgeons and is always considered when physicians treat critically ill infants with abdominal symptoms and unknown diagnoses.

Malrotation has primarily diagnosed in early childhood, with an estimated onset of symptoms during the first year of life in $90 \%$ of the cases ${ }^{4-6}$. There are recent reports of manifestation later in life, both as emergency conditions or more chronic 
gastrointestinal symptoms ${ }^{2,4,7}$. The exact incidence of intestinal malrotation is thus still difficult to determine. It was earlier described to be approximately $0.2 \%^{5,8,9}$, but an incidence of up to $1 \%$ has been reported $^{10}$. Improved radiological facilities, including multi-detector CTscans, provides new possibilities to identify anatomical aberrations.

The aim of this study is to assess adult intestinal malrotation regarding the methods of investigation and surgical management.

\section{Patients and Methods}

Three patients with adult intestinal malrotation were assessed regarding presentation, investigation, management and post operative complications

\section{The first case:}

A 30 year old female patient presented with history of repeated vomiting and weight loss. On examination, she was dehydrated and malnourished. Laboratory investigations revealed hypokalemia and hypoalbuminemia, OGD was also normal. A CT scan revealed a clear sign of intestinal malrotation with twisting and obstruction of the duodenum (Whirlpool sign) with hugely distended stomach and proximal duodenum. The patient was admitted to the hospital, fluid and electrolyte were corrected together with introduction of NG tube. Prophylactic antibiotic and anticoagulant were started.

To establish the degree of malrotation, the radiologist identified the position of the duodenum, the proximal small bowel, the location of the caecum and the orientation of the mesenteric vessels using intravenous per oral as well as intrarectal contrast (triple-contrast). Twisting of the mesentery of the small bowel, the "whirlpool-sign", typical for a volvulus was noted. These findings were also re-scrutinized and confirmed independently by one dedicated radiologist.

Investigations utilizing US and multidetector computer tomography were used. A "whirlpool-sign" signifying the presence of rotation of the bowel was detected with huge dilatation of the stomach \& duodenum (figures 1-3).
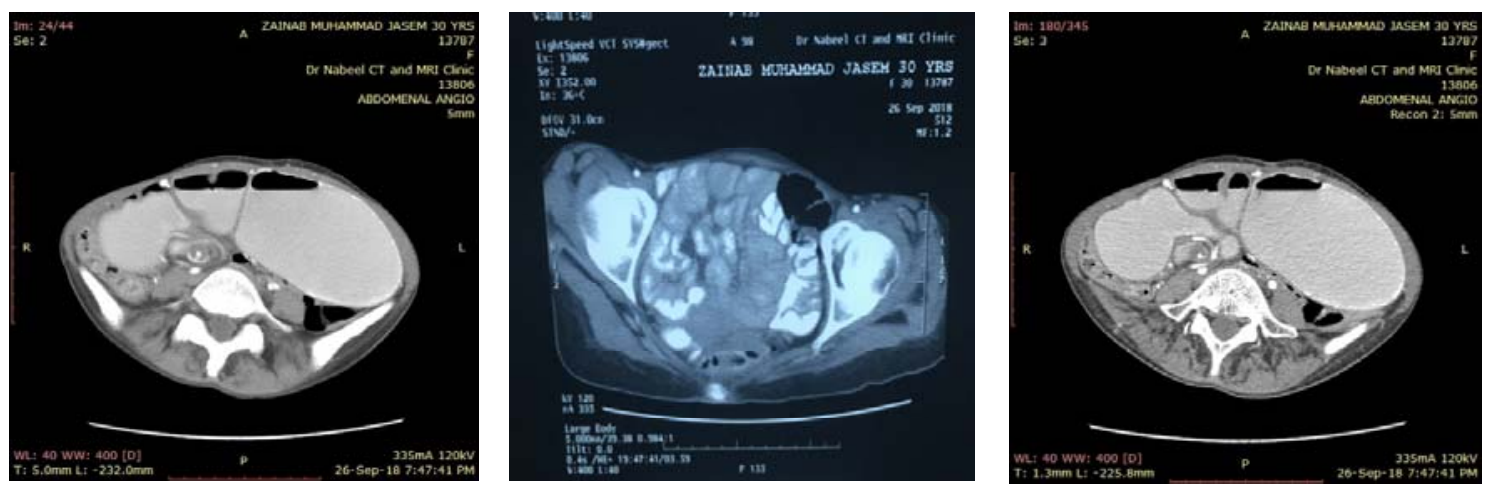

Fig. 1: Multi-detector computer tomography, A whirlpool sign signifying a presence of bowel rotation.
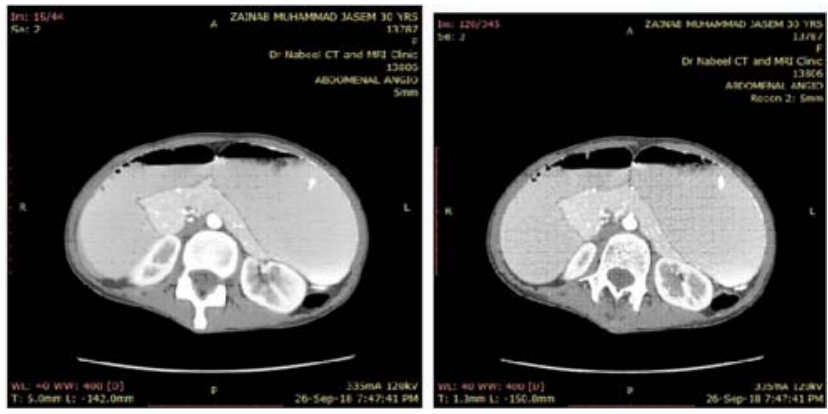

Fig. 2: Dilated proximal duodenum on CT scan. 


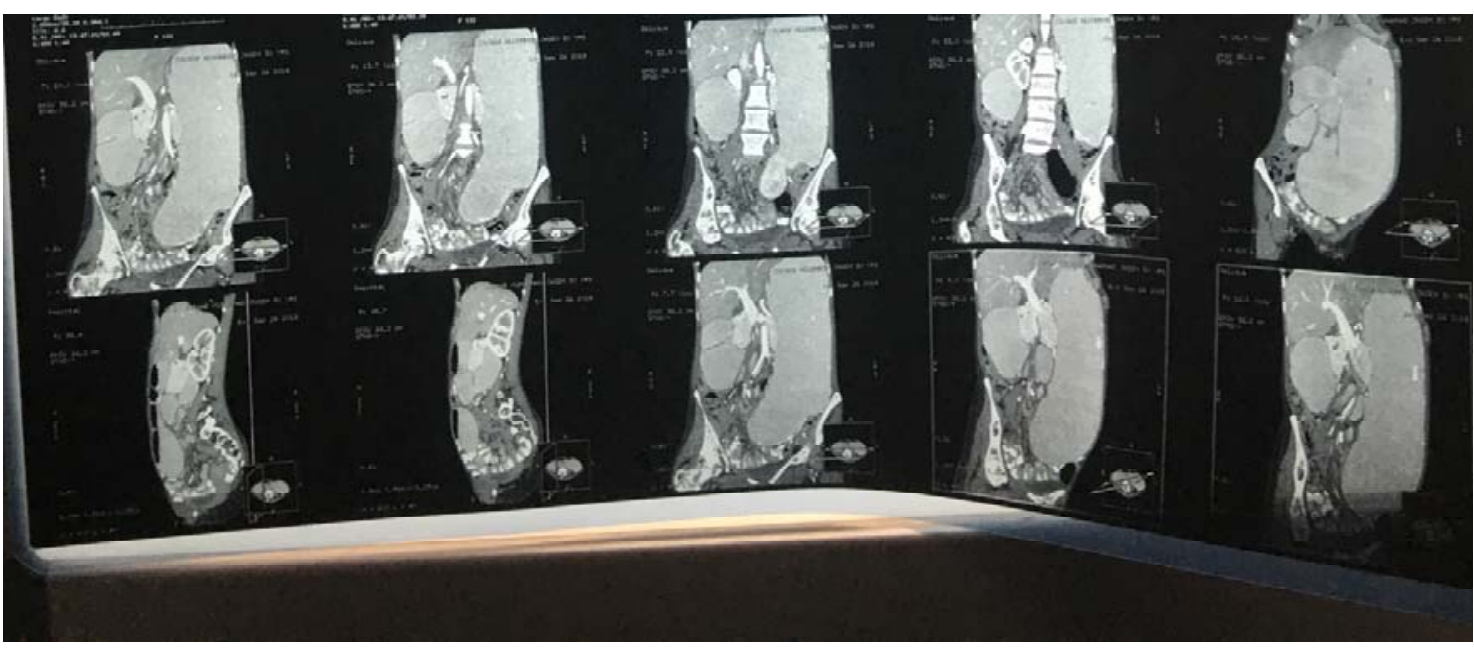

Fig.3: Hugely dilated stomach reach pelvis

A decision for surgery was made. Intraoperatively, malrotation was treated by corrective surgery according to the technique originally described by Ladd. The intestines were de-rotated in a counter clockwise manner and the Ladd band was carefully dissected and removed, the duodenum was fully mobilized and the obstruction was relived. The mesentery was broadened and the adhesions surrounding the mesenteric vessels were dissected in order to avoid future recurrence of the volvulus. After that, the small bowel was placed to the right and the colon to the left side of the abdominal cavity in a "non-rotational" position.

Gastro-jejnostomy was done for the hugely distended stomach to avoid risk of gastroparesis postoperatively (fig.4).

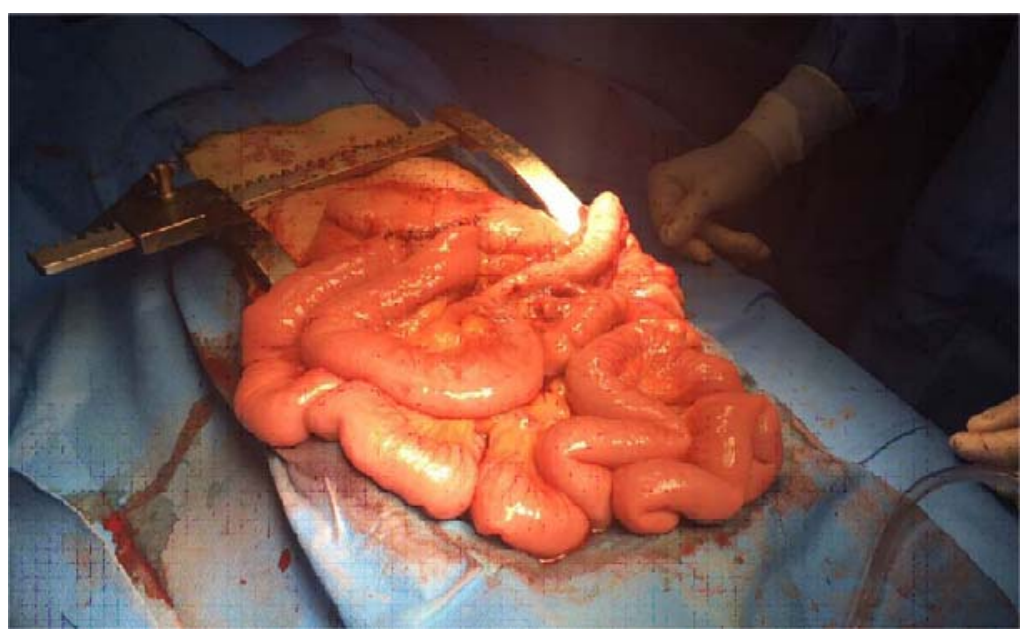

Fig.4: Small bowel on the right and large bowel on left with gastro- jejnostomy

Appendectomy was performed during operation for malrotation for two reasons: The normal anatomical placement of the appendix is disrupted when the cecum is placed in the left upper quadrant, making the diagnosis of future appendicitis challenging. Dissection of the peritoneal bands can cause damage to the appendiceal vessels. The patient was assessed in the outpatient department in 2 weeks, 1 months, 2 years after surgery. During follow-up, questions were focused on remaining intense or chronic pain, postprandial nausea, vomiting and constipation. Patients were also asked if they are satisfied with the improvement of their general physical condition. 


\section{The second case:}

A 47 year old male, presented with chronic abdominal pain (post prandial), and vomiting. On examination, the

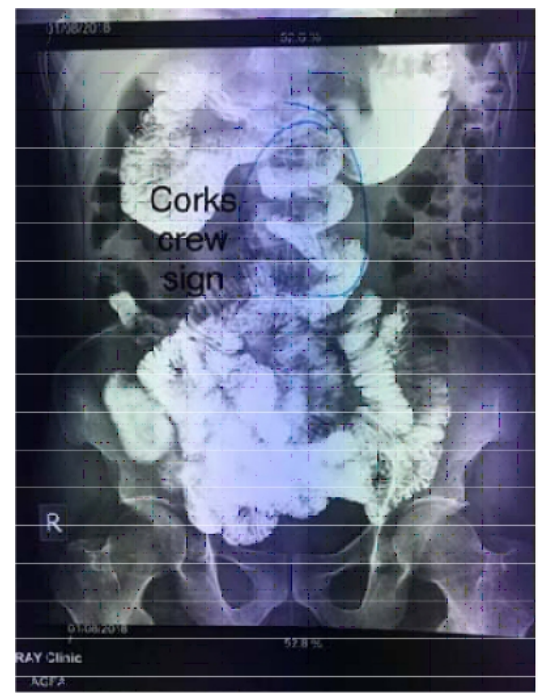

abdomen was slightly distended with mild tenderness. Barium follow through revealed corkscrew sign (sign of intestinal malrotation), fig.5.

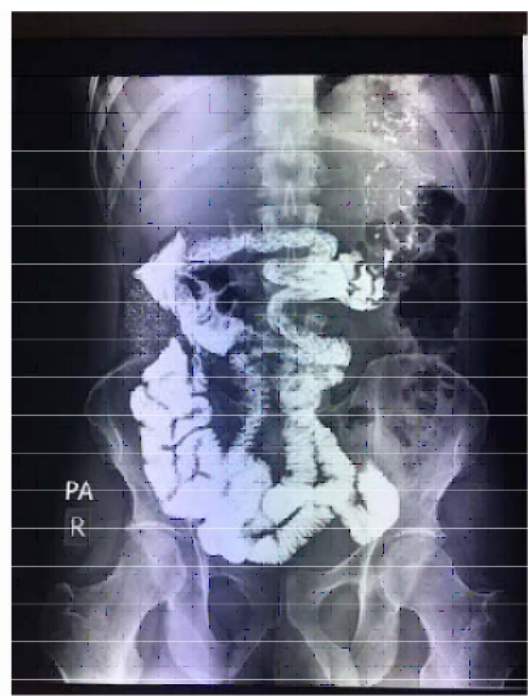

Fig.5: Barium follow through show intestinal malrotation.

Decision for surgery was taken and at operation, the intestine was de-rotated in a counter clockwise manner and all Ladd's bands were carefully dissected and removed, the duodenum was fully mobilized (Kocharization) and the obstruction was relived, fig.6.
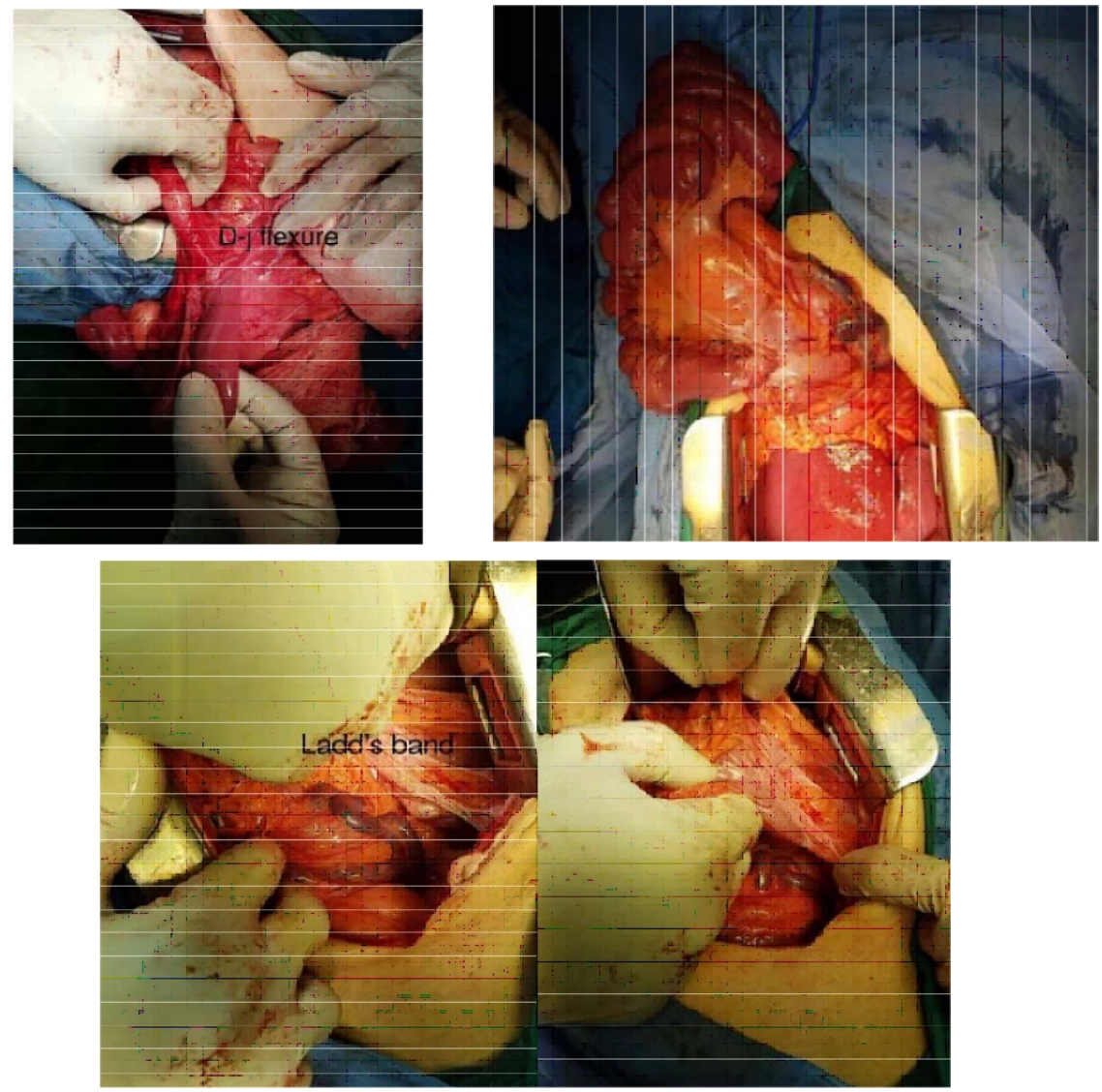

Fig.6: Intestinal malrotation.and ladd's band 
Appendectomy was performed during operation for this case as shown in fig.7.
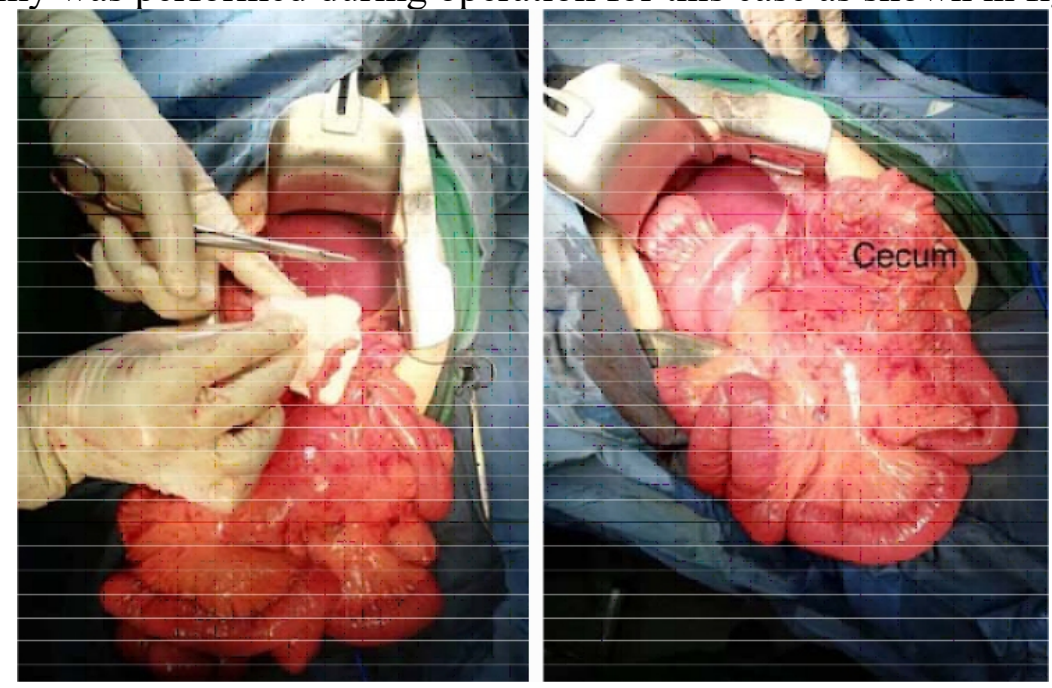

Fig.7: Appendectomy \& after de-rotation of intestinal malrotation.

\section{The third case:}

A 25 year old 8 missed periods pregnant patient came with elevated liver enzymes and low platelets count without any history of abdominal pain or vomiting. The U/S and CT scan revealed liver hematoma with co-accidental finding of a "whirlpool-sign" signifying a presence of

bowel rotation, fig.8. A diagnosis of hemolysis, elevated liver enzymes, and a low platelet count (HELLP) syndrome was made together with intestinal malrotation. Patient was treated conservatively for few days and discharged well from the hospital.
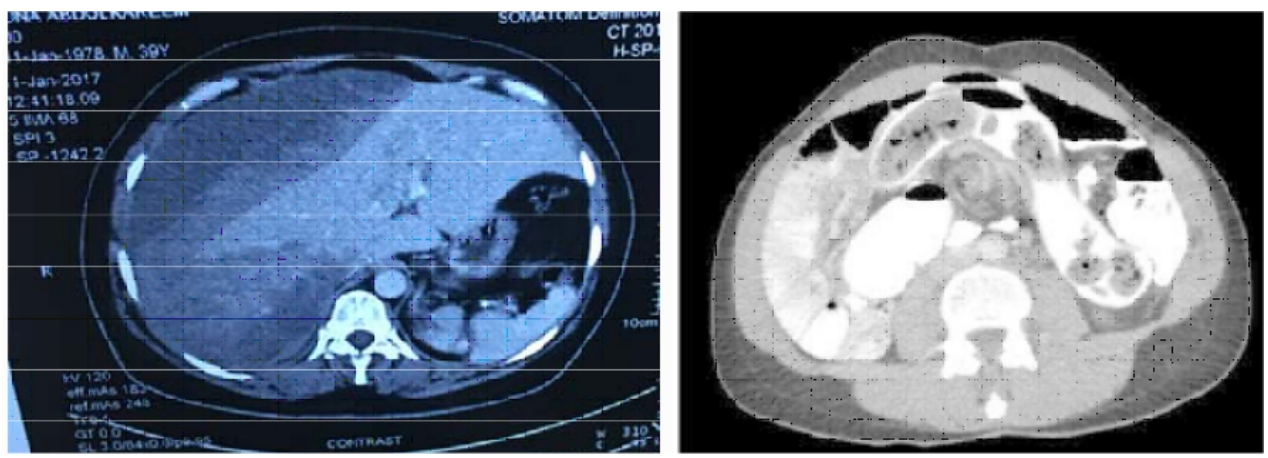

Fig.8: Liver hematoma and incidental intestinal malrotation.

\section{Results}

Patients participating in this study were three, two of them were females and one male (2:1), two of them presented with sign and symptoms of intestinal

obstruction. Two cases were diagnosed by CT scan (whirlpool-sign) and one case by GIT series (corkscrew sign) as demonstrated in table I.

Table I: Type of presentation and methods of diagnosis

\begin{tabular}{|l|l|l|}
\hline \multirow{3}{*}{ Presentation } & Type & Number \\
\cline { 2 - 3 } Methods of diagnosis & Intestinal obstruction & 2 \\
\cline { 2 - 3 } & CT Scan & 2 \\
\cline { 2 - 3 } & GIT Series & 1 \\
\hline
\end{tabular}


Two cases were treated surgically (Ladds procedure) and had an uneventful postoperative course, leaving the hospital within 5 to 10 days. One case was treated conservatively. No patient had intestinal resection and no mortality reported in this study, table II.

Table II: types of treatment

\begin{tabular}{|l|l|}
\hline Treatment type & No. \\
\hline Conservative treatment & 1 \\
\hline Ladd's surg. procedure & 2 \\
\hline Resection small intestine & 0 \\
\hline
\end{tabular}

\section{Discussion}

Congenital intestinal malrotation should be recognized as a reason for abdominal pain in adults which has also been emphasized in a recent population based study by Coe et $\mathrm{al}^{11}$. We describe a substantial number of symptomatic patients being diagnosed in mature age often after several years of suffering. This study showed that young adults have a tendency towards more severe symptoms requiring emergency treatment. No statistical comparison has been made with patients suffering adhesive bowel obstruction from other reasons, and thus age and concurrent developmental disorders are the only markers identified necessitating increased awareness when considering malrotation as a cause for obstruction with severe symptoms ${ }^{12}$.

In this clinical study, the patients experienced a considerable improvement in their general status after surgical intervention. Nehra presents an excellent retrospective study which includes 130 patients of all ages treated at a single institution $^{7}$. Only $30 \%$ of the patients were below 1 year of age, and as many as $48 \%$ were above 18 years of age at the time of diagnosis. They described a decreased risk for volvulus with age, which also was confirmed among adult cases in the present study.

The increased recognition of intestinal malrotation in adults may be explained by the more frequent use of abdominal CTscan and barium study refinements of methods that more correctly visualize variations in the abdominal anatomy ${ }^{4,13}$.
A multi-detector CT-scan provides the possibility of following the exact course of the duodenum as well as the position of the small bowel and the caecum. Importantly, the orientation of the superior mesenteric vessels also becomes assessable, sometimes with an additional depicted rotation of the mesentery of the bowel forming a "whirlpool-sign". This may indicate a precarious circulation of the bowel, possibly requiring rapid surgical intervention.

In children a contrast of the stomach and small intestine is usually enough to diagnose malrotation where the displacement of duodenum is clearly shown. In adults, where other reasons for intestinal obstruction are more frequent, a more detailed imaging including exact criteria prior to surgery is valuable $\mathrm{e}^{12}$.

In this study, one third of the patients were operated as emergency, compared to the higher incidence of $75 \%$ reported in pediatric series ${ }^{14}$. Many patients had ongoing abdominal discomforts since childhood, while others encountered a relatively sudden onset of symptoms leading to chronic episodes of abdominal pain. A considerable proportion of the patients in this series had reached a high age before being informed of their abnormality. Gastroenterologists and surgeons treating adults probably put less emphasis on the possibility of a congenital malformation causing the abdominal symptoms ${ }^{7,15}$. Intestinal malrotation may have a "syndromal" appearance and is often accompanied by 
other anomalies (30-80\%), including developmental disorders of the $\mathrm{CNS}^{2,15}$. It is important to have a vigilant strategy for malrotation when investigating abdominal complaints in mentally disabled patients who lack the possibility to describe their symptoms. The comorbidity caused by these concurrent disorders may be one reason for the high mortality during follow-up, since only one patient died from complications after surgery in terms of short bowel after resection of ischemic intestine ${ }^{12}$.

In pediatric reports, the recurrence rate after Ladd's procedure is considered low with a reported incidence between 2 and $7 \%{ }^{14,16}$. The higher recurrence rate reported here is partly explained by a learning curve among the involved surgeons, but more long-lasting preoperative symptoms may also add to the complexity of surgical problems. Interestingly, it has been shown that also children operated upon later during childhood have a higher incidence of reoperation ${ }^{17}$.
In our study one case with a radiological malrotation diagnosis have not yet undergone surgery, claiming that they currently experience only mild symptoms and wish for a conservative approach. Today, many authors advocate surgical correction of malrotation due to the difficulty in predicting who will be striked by torsion of the midgut, bringing an urgent, life-threatening condition in the future ${ }^{12}$. Furthermore, one cannot be certain that patients without complaints are truly free from symptoms ${ }^{18,19}$.

In conclusion, Intestinal malrotation should be regarded as a malformation affecting all age groups since it is obviously more common in the adult population. A properly performed contrast enhanced computer tomography reveals the malformation and enables surgical treatment in adults with a history of long-periods of abdominal complaints. In addition, and most importantly, acute obstruction with volvulus occurs in all ages and needs emergency surgery.

\section{References}

1. Dott NM (1923) Anomalies of intestinal rotation: their embryology and reports from five cases. Br J Surg 11:251- 286View ArticleGoogle Scholar

2. Penco JM, Murillo JC, Hernàndez A, De La Calle Pato U, Masjohan DF, Aceituno FR (2007) Anomalies of intestinal rotation and fixation: consequences of late diagnosis beyond two years of age. Pediatr Surg Int 23:723-732View ArticleGoogle Scholar

3. Ladd W (1936) Surgical disease in the alimentary tract in infants. N Engl J Med 215:705-708View ArticleGoogle Scholar

4. Pickhardt PJ, Bhalla J (2002) Intestinal malrotation in adolescents and adults: spectrum of clinical and imaging features. AJR 179:1429-

1435View ArticleGoogle Scholar

5. Stewart DR, Colodny AL, Daggett WC (1976) Malrotation of the bowel in infants and children: a 15 year review. Surgery 79(6):716720Google Scholar

6. Vaos G, Misiakos EP (2010) Congenital anomalities of the gastrointestinal tract diagnosed in adulthood—diagnose and management. J Gastrointest Surg 14:916-925View ArticleGoogle Scholar

7. Nehra D, Goldstein AM (2011) Intestial malrotation: varied clinical presentation from early infancy through adulthood. Surgery 149(3):386-393View ArticleGoogle Scholar

8. Donnellan WL, Kimura K (1996) Malrotation, intestinal hernias, congenital band. In: Donnellan WL (ed) Abdominal surgery of infancy and childhood. Harwood Academic, Austria-United States, pp 1-27Google Scholar

9. Clark LA, Oldham KT (2002) Malrotation. In: Ashcraft KW, Murphy JP, Sharp RJ, Sigalet DL, Snyder CL (eds) Pediatric surgery, 3rd edn. WB Saunders, Philadelphia, pp 425-434Google Scholar

10. Adams SD, Stanton MP (2014) Malrotation and intestinal atresias. Early Hum Dev 90(12):921-925 (review)View ArticleGoogle Scholar 11. Coe T, Chang D, Sicklick J (2015) Small bowel volvulus in the adult populace of the United States: results from a population-based study. Am J Surg 210(2):201-210.e2. doi:10.1016/j.amjsurg.2014.12.048 (epub 2015 Apr 30)

12. Britt Husberg,Karin Salehi (2016) Congenital intestinal

malrotation in adolescent and adult patients: a 12-year clinical and radiological survey.

13. Emanuva OF, Ayantunde A, Davies TW (2011) Midgut malrotation first presenting as acute bowel obstruction in adulthood: a case report and literature review. World J Emerg Surg 6:22View ArticleGoogle Scholar

14. El-Gohary Y, Alagtal M, Gillick J (2010) Long-term complications following operative intervention for intestinal malrotation: a 10-year review. Pediatr Surg Int 26:203-206View ArticleGoogle Scholar

15. Nagdeve NG, Qureshi AM, Bhingare PD, Shinde SK (2012) Malrotation beyond infancy. J Pediatr Surg 47:2026-2032View ArticleGoogle Scholar

16. Freitz R, Vos A (1997) Malrotation: the postoperative period. J Pediatr Surg 32(9):1322-1324View ArticleGoogle Scholar

17. Durkin ET, Lund DP, Shaaban AF, Schurr MJ, Weber SM (2008) Agerelated differences in diagnose and morbidity in intestinal malrotation. J Am Coll Surg 206:658-663View ArticleGoogle Scholar

18. Raitio A, Green PA, Fawkner-Corbett DW, Wilkinson DJ, Baillie CT (2015) Malrotation: age-related differences in reoperation rate. Eur J Pediatr Surg (epub ahead of print)Google Scholar

19. Moldrem AW, Papaconstantinou H, Broker H, Megison S, Jeyarajah DR (2008) Late presentation of intestinal malrotation: an argument for elective repair. World J Surg 32:1426-1431View ArticleGoogle Scholar 